\title{
Vasculitis leucocitoclásticas como manifestación extraintestinal dermatológica rara de la enfermedad inflamatoria intestinal asociada con Clostridium: reporte de un caso
}

\section{Leukocytoclastic vasculitis as a rare extraintestinal dermatologic manifestation of inflammatory bowel disease associated with Clostridium difficile: Case report}

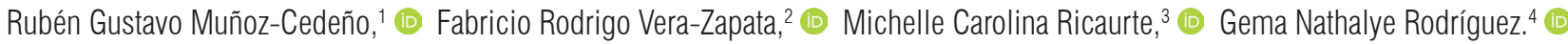

\author{
GaCCESO ABIERTO \\ Citación: \\ Muñoz-Cedeño RG, Vera-Zapata FB, Ricaurte MC, \\ Rodríguez GN. Vasculitis leucocitoclásticas como \\ manifestación extraintestinal dermatológica rara \\ de la enfermedad inflamatoria intestinal asociada \\ con Clostridium: reporte de un caso. Rev Colomb \\ Gastroenterol. 2021;36(Supl.1):12-18. https://doi. \\ org/10.22516/25007440.500
}

Médico general, posgrado en Gastroenterología Hospital de Especialidades Dr. Abel Gilbert Pontón. Guayaquil, Ecuador

Médico gastroenterólogo, Hospital General de Guayaquil los Ceibos (IESS). Guayaquil, Ecuador.

Médico general residente, Hospital de Especialidades Dr. Abel Gilbert Pontón. Guayaquil, Ecuador.

${ }^{4}$ Médico general residente, Hospital Universitario

de Guayaquil. Guayaquil, Ecuador.

*Correspondencia: Rubén Gustavo Muñoz. drubenmunoz@gmail.com

Fecha recibido: $\quad 15 / 01 / 20$ Fecha aceptado: 21/07/20

\section{Resumen}

Las vasculitis leucocitoclásticas se definen como el daño e inflamación de las paredes vasculares, son aquellas vasculitis de pequeños vasos que anatomopatológicamente presentan leucocitoclasia y puede observarse como una manifestación extraintestinal de la enfermedad inflamatoria intestinal. En la colitis ulcerativa se presentan en menor frecuencia, por inmunocomplejos generados en la mucosa intestinal debido a la exposición del tejido linfoide submucoso a antígenos fecales; podrían precipitarse en las paredes de los pequeños vasos. Se pueden asociar con Clostridium difficile, que es un bacilo grampositivo esporulado, anaerobio estricto, que se encuentra normalmente en el medio ambiente y produce colitis, que se manifiesta como un cuadro diarreico presentado después de la ingesta de antibióticos y altera la flora bacteriana común de este órgano. El caso se trata de un paciente 36 años de edad con cuadro de diarreas líquidas con moco y escaso sangrado; se realizó un estudio endoscópico y anatomopatológico en el que se observó colitis ulcerativa con coproparasitario positivo para antígeno de $C$. difficile, y en su hospitalización presentó lesiones dérmicas petequiales y necróticas en el cuarto dedo de la mano izquierda, que en la biopsia dio como resultado vasculitis de pequeños vasos. En este artículo se revisan de forma práctica los aspectos relacionados con la fisiopatología, histología, tratamiento y diagnósticos de la manifestación extraintestinal dermatológica rara, como la vasculitis leucocitoclástica en pacientes con colitis ulcerativas asociadas con Clostridium.

\section{Palabras clave}

Vasculitis leucocitoclásticas, manifestaciones extraintestinales, Clostridium difficile, colitis ulcerativa, enfermedad inflamatoria intestinal.

\begin{abstract}
Leukocytoclastic vasculitis is defined as the damage and inflammation of the vascular walls. The term refers to vasculitis of the small vessels that anatomopathologically present leukocytoclasia and it can be seen as an extra-intestinal manifestation of inflammatory bowel disease. In ulcerative colitis, it occurs less frequently due to immune complexes produced in the intestinal mucosa by exposure of the submucosal lymphoid tissue to fecal antigens, which could precipitate in the walls of the small vessels. This condition can be associated with Clostridium difficile, which is a gram-positive, sporulated, strict anaerobic bacillus, normally found in the environment. It causes colitis that manifests as a diarrheal disease following the ingestion of antibiotics that alter the common bacterial flora of this organ. This is the case report of a 36-year-old patient with liquid diarrhea with mucus and scarce bleeding. Endoscopic and anatomopathological studies were performed, finding ulcerative colitis with positive coproparasite for Clostridium difficile antigen. The patient was hospitalized, and during his stay, he presented with petechiae and necrotic skin lesions on the fourth finger of the left hand. Skin biopsy showed small vessel vasculitis. This article is a practical review of the pathophysiology, histology, treatment, and diagnosis of a rare dermatologic extraintestinal manifestation, namely, leukocytoclastic vasculitis, in patients with C. difficile-associated ulcerative colitis.
\end{abstract}

\section{Keywords}

Leukocytoclastic vasculitis; Extraintestinal manifestations; Clostridium difficile; Ulcerative colitis; Inflammatory bowel disease. 


\section{INTRODUCCIÓN}

Las vasculitis leucocitoclásticas se definen como el daño e inflamación de las paredes vasculares, son aquellas vasculitis de pequeños vasos que anatomopatológicamente presen$\tan$ leucocitoclasia o vasculitis por hipersensibilidad (1). Es una manifestación extraintestinal de la enfermedad inflamatoria intestinal, que está presente en colitis ulcerativa (CU) en un $15 \%$ a $20 \%$, ya que tienen un mecanismo etiopatogénico común que se relacionan por inmunocomplejos generados en la mucosa intestinal debido a la exposición del tejido linfoide submucoso a antígenos fecales; podrían precipitarse en las paredes de los pequeños vasos y clínicamente pueden ser sincrónicas en su aparición. Se han reportado hasta el 2014 en la literatura inglesa al menos 20 casos con predomino de edad de 40 años $(2,3)$. La asociación con Clostridium difficile puede alterar el curso de la enfermedad al activar la respuesta inmunitaria hacia el patógeno que causa la infección que se encuentra normalmente en el medio ambiente y produce colitis, que se manifiesta como un cuadro diarreico presentado después de la ingesta de antibióticos que altera la flora bacteriana común de este órgano (4).

\section{PRESENTACIÓN DEL CASO}

Se trata de un paciente masculino de 36 años de edad, sin antecedentes patológicos personales, con antecedentes familiares (la madre falleció por cáncer de colon); presentó un cuadro clínico de inicio de 5 años de evolución por diarreas líquidas con moco y escaso sangrado manejado con antibiótico por varias ocasiones con ciprofloxacina 500 mg vía oral por 7 días y probióticos. Ingresó al centro de salud por una agudización de su cuadro clínico hace 20 días por dolor abdominal tipo cólico acompañado con distención abdominal, diarrea líquida con moco y sangre de 4 a 6 deposiciones al día, alza térmica no cuantificada, artralgia $\sin$ artritis y pérdida de peso aproximado de $10 \mathrm{~kg}$ en 1 año. En el examen físico se observó abdomen blando depresible con eritema, edema en ambas manos y pies, y presencia de aftas bucales en la lengua (Figuras 1 y 2 ).

En los exámenes de ingreso se reportó hemoglobina: 7,7 $\mathrm{mg} / \mathrm{dL}$; hematocrito: $26,5 \%$; leucocitos: $11830 / \mathrm{mm}^{3}$; neutrófilos de 75,4\%; plaquetas: 544 000; PCR: $16,17 \mathrm{mg} /$ $\mathrm{dL}$; electrolitos y función renal normal; prueba de VDRL no reactiva; vitamina $B_{12}>2000$; ferritina: 170,58 ; hierro sérico: 31; transferrina: 205; marcadores tumorales dentro de los valores normales; serología para citomegalovirus, virus de herpes, rubeola, virus de hepatitis $\mathrm{B}$, virus de hepatitis $\mathrm{C}$ y virus de inmunodeficiencia humana (VIH) negativos; coproparasitario toxina de $C$. difficile positivo para antígeno; cultivo de heces negativo y, de los marcadores inmunológi- cos, solo dio positivo el anticuerpo anticitoplasma de neutrófilos (c-ANCA); el resto, negativos. Se realizó un estudio endoscópico de las vías digestivas altas y se encontraron úlceras en el esófago distal (Figura 3), se tomó biopsia tanto de los bordes como del centro de las úlceras para realizar diagnósticos diferenciales (citomegalovirus, herpes simples, enfermedad de Crohn, entre otros), en la cual se reportó infiltrado inflamatorio mixto con polimorfos nucleares en el epitelio, inmunohistoquímica para citomegalovirus y herpes negativo. A nivel prepilórico, se encontró engrosamiento de los pliegues (Figura 4) y se tomó una biopsia para descartar el diferencial de la enfermedad inflamatoria intestinal con enfermedad de Crohn, que reportó gastritis crónica activa erosiva con GIEMSA y colonización hipodensa de bacilos Helicobacter pylori, que se relacionó con lesiones fortuitas. En la colonoscopia de todo el colon se observó una alteración del patrón vascular, eritematoso, granular, edematoso y friable; en el colon descendente se observó una lesión pseudopolipoidea (Figura 5), y en la biopsia se encontró CU.

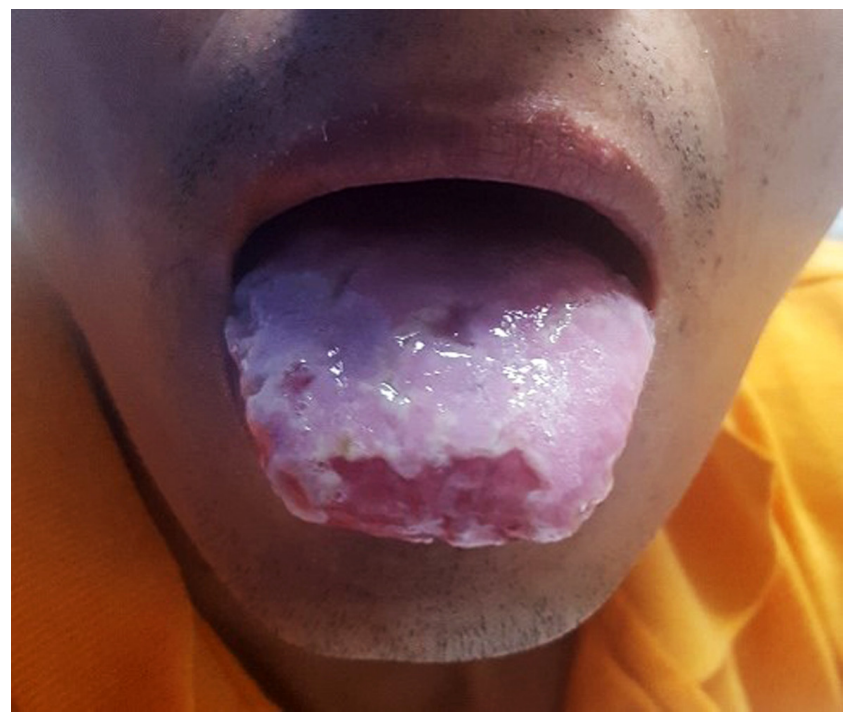

Figura 1. Presencia de aftas bucales en la lengua.

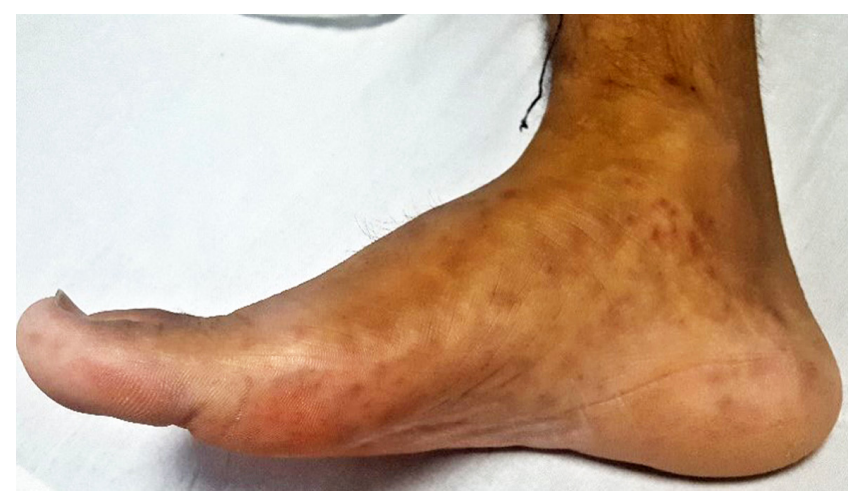

Figura 2. Lesiones eritematosas en la parte interna del pie derecho. 


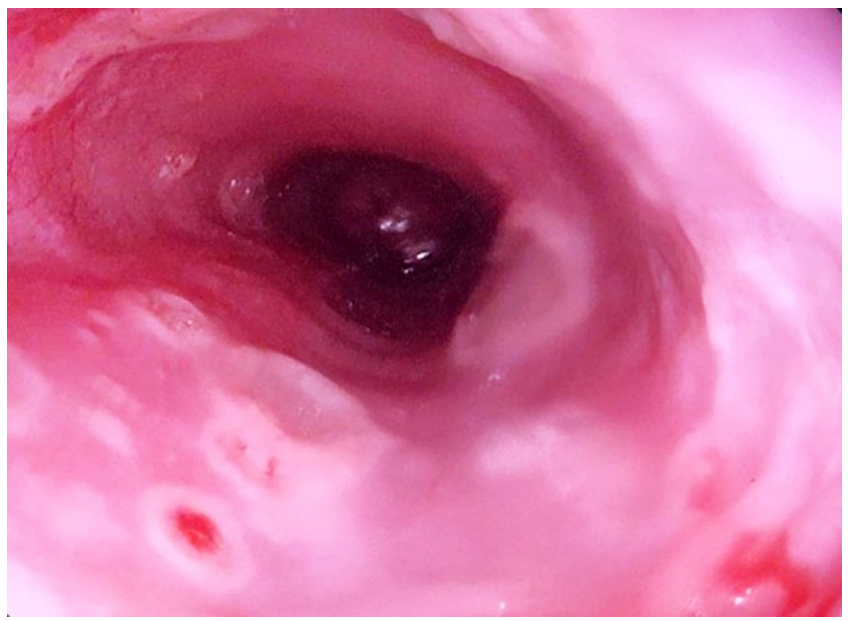

Figura 3. Videoendoscopia alta en la que se muestra la presencia de úlceras de $5 \mathrm{~mm}$ con bordes sobreelevados con fondo eritematoso en el esófago distal.

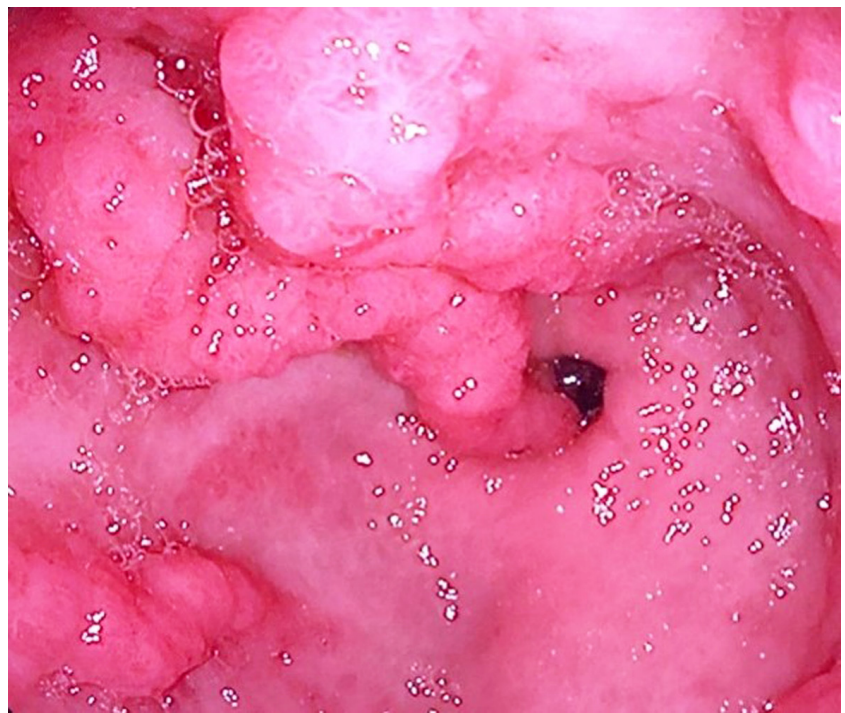

Figura 4. Videoendoscopia alta a nivel de antro en la que se muestra la presencia de engrosamiento de los pliegues prepilóricos con presencia de eritema y edema.

48 horas después del procedimiento endoscópico presentó lesiones dérmicas petequiales y necróticas en el cuarto dedo de la mano izquierda (Figuras 6 y 7 ), por lo que se le realizó una ecografía Doppler de miembro superior en la que se encontraron fluidos disminuidos con ondas parvus tardus en las arterias digitales palmares y dorsales del cuarto dedo de la mano izquierda (Figura 8). Fue valorado por dermatología y se realizó la toma de biopsia que dio como resultado vasculitis de pequeños vasos (paniculitis septal, infiltrado inflamatorio perivascular con predominio de neutrófilos, daño fibrinoide y hematíes extravasados). Recibió tratamiento de 3 pulsos de metilprednisolona 500 mg intravenoso, que posteriormente pasó a ser por vía oral con prednisona $60 \mathrm{mg}$ hasta decalar y se retiró el corticoide; posteriormente se administró metronidazol $500 \mathrm{mg}$ intravenoso por 14 días y mesalazina $3 \mathrm{~g}$ por vía oral hasta el momento. Actualmente, el paciente se mantiene asintomático sin signos de lesiones en las manos ni la lengua, y en remisión clínica de la $\mathrm{CU}$, sin uso de corticoides, con mesalazina $3 \mathrm{~g} /$ día y tiene controles en consulta externa sin manifestaciones clínicas.

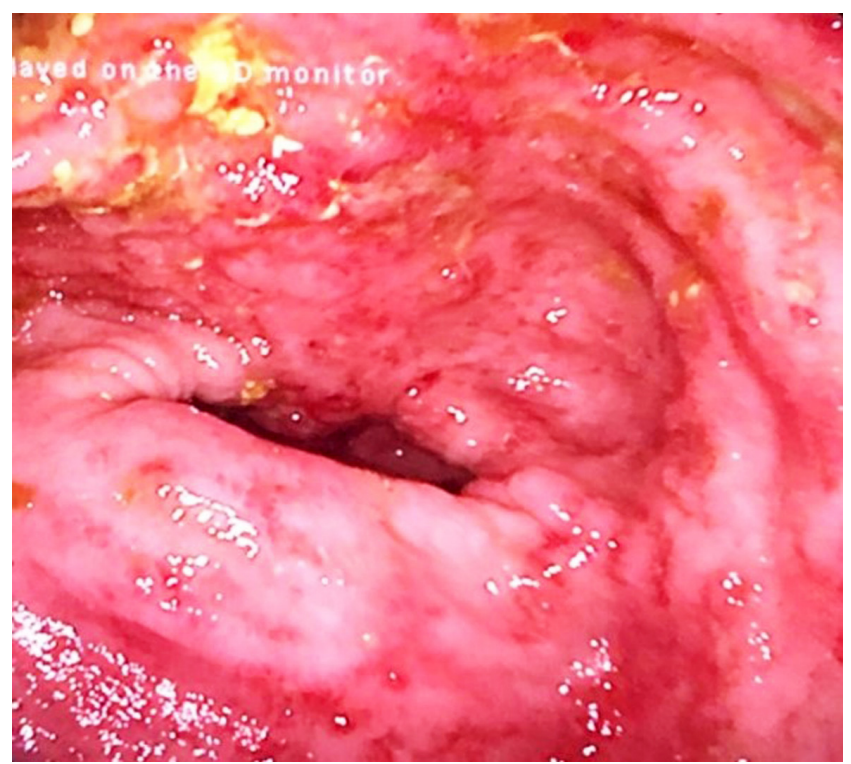

Figura 5. Colonoscopia a nivel todo el colon en la que se observa alteración del patrón vascular, mucosa, eritema granular y edema friable con presencia de úlceras superficiales a nivel del colon sigmoide.

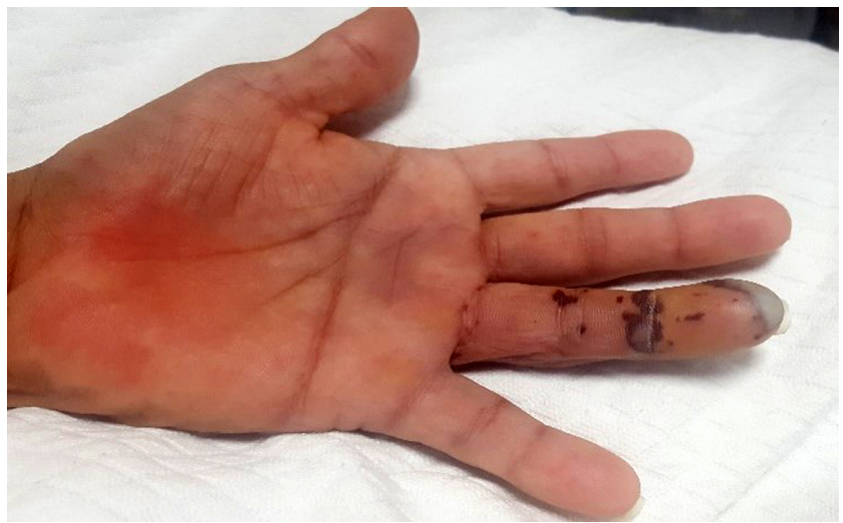

Figura 6. Lesiones dérmicas petequiales y necróticas en el cuarto dedo de la mano izquierda.

\section{DISCUSIÓN}

La enfermedad inflamatoria intestinal tiene múltiples manifestaciones extraintestinales entre ellas las manifestaciones cutáneas en un 15\%, y otras más frecuentes que incluye afectación osteoarticular (osteopenia, osteoporo- 


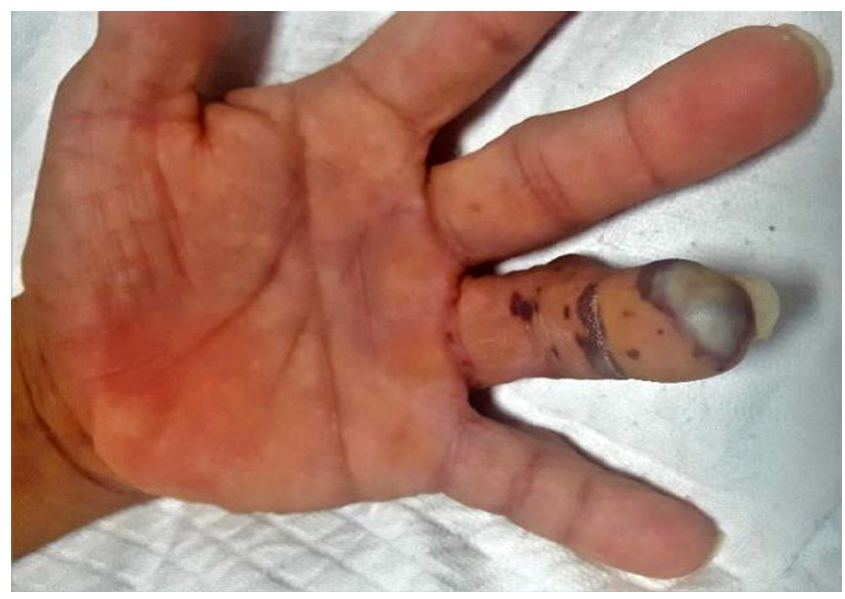

Figura 7. Lesión necrótica con área blanquecina en la parte distal del cuarto dedo de la mano izquierda.

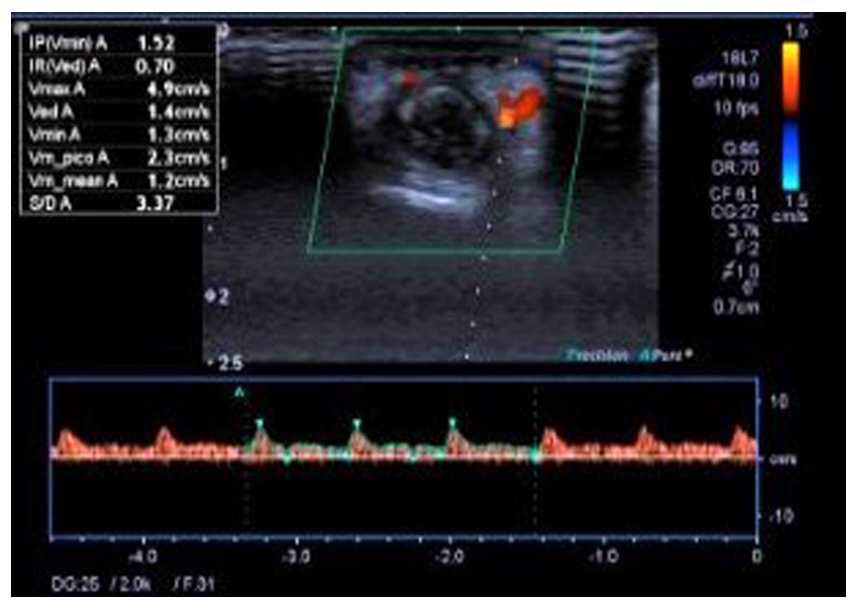

Figura 8. Ecografía Doppler del miembro superior, fluidos disminuidos con ondas parvus tardus en las arterias digitales palmares y dorsales del cuarto dedo de la mano izquierda.

ciona con artritis reumatoide, lupus eritematoso sistémico, síndrome fosfolipídico, síndrome de Sjögren y enfermedad de Behçet; también tiene un mecanismo etiopatogénico común con la $\mathrm{CU}$ y, por tanto, la relación causal de los dos procesos se da por inmunocomplejos generados en la mucosa intestinal por exposición del tejido linfoide submucoso a antígenos fecales; podrían precipitarse en las paredes de los pequeños vasos y activarían citocinas proinflamatorias, como el sistema de la interleucina 15 (IL-15) y su receptor; así como podrían diferenciar las células B de la lámina propia con la formación de las lesiones típicas en pacientes con CU establecida ( $58 \%$ ) como manifestación extraintestinal $(1,3,10,11)$.

Su presentación clínica de vasculitis leucocitoclástica se caracteriza por púrpura palpable, con síntomas de dolor y ardor en múltiples áreas (58 \%) o en un solo sitio (42\%). Sus lesiones se localizan predominantemente en las extremidades inferiores $(83 \%)$, seguidas de las extremidades superiores $(42 \%)$, el tronco $(25 \%)$ y los glúteos $(25 \%)$ $(3,12,13)$. Ante la sospecha de vasculitis leucocitoclástica como manifestación extraintestinal dermatológica de $\mathrm{CU}$, se debe realizar una biopsia cutánea para observar las características histológicas de infiltración de neutrófilos y linfocitos alrededor de los vasos sanguíneos dérmicos que muestran leucocitoclasia, depósito de fibrina en los vasos, trombos de fibrina, extravasación de eritrocitos y necrosis epidérmica resultante $(10,14)$.

Se consideran como síntomas en los pacientes con vasculitis leucocitoclástica y CU la fiebre, mialgias, artralgias, hematuria, dolor abdominal, vómitos, deposiciones diarreicas sanguinolentas, incontinencia rectal y tenesmo; otros síntomas 
incluyen anorexia, astenia y pérdida de peso. Se reporta que la aparición de síntomas se da aproximadamente a la semana de la exposición al factor detonante $(9,15,16)$. La afectación colónica en pacientes con vasculitis leucocitoclástica y CU se observa en el $50 \%$ con pancolitis, en el $40 \%$ con colitis distal y en el $10 \%$ con desarrollo de vasculitis leucocitoclástica después de una colectomía total (2).

Como diagnóstico diferencial, se deben incluir otras causas de manifestaciones extraintestinales dermatológicas y de enfermedad inflamatoria intestinal que deben ser descartadas antes de un diagnóstico definitivo. Dentro de las dermatológicas se incluyen pioderma gangrenoso, vasculitis necrosante, poliartritis nodosa cutánea y vasculitis granulomatosa; mientras que en las infecciosas se encuentran C. difficile, Escherichia coli, Salmonella, Shigella, Yersinia y Campylobacter. Otros diagnósticos diferenciales son enfermedad de Crohn, colitis pseudomembranosa y síndrome de intestino irritable $(9,16,17)$.

La asociación de $C$. difficile en pacientes con vasculitis leucocitoclástica y CU se debe a que es un bacilo grampositivo esporulado, anaerobio estricto. Se encuentra normalmente en el medio ambiente. $\mathrm{Al}$ causar una infección en el ser humano, generalmente produce colitis que se manifiesta como un cuadro diarreico, fiebre, anorexia, reacción leucemoide, hipoalbuminemia y sangrado posterior a la ingesta de antibióticos que altera la flora bacteriana común de este órgano (18). Es la causa de diarrea más común en el ambiente hospitalario, debido a su asociación etiológica con antibióticos. El riesgo de desarrollar la infección depende además de otros factores como edad, dieta, dosis del antibiótico causante administrado, entre otros (19).

El diagnóstico se basa en criterios clínicos y de laboratorio. Dichos criterios deben incluir el criterio clínico de 3 deposiciones diarreicas de inicio súbito sin causa identificada, acompañado de toxinas A o B en las heces, o la presencia de pseudomembranas en el colon detectadas mediante un procedimiento endoscópico (18). En un estudio de Zacharioudakis y colaboradores en Estados Unidos se estudiaron los factores que se han determinado como desencadenantes de contraer la infección o la transición de colonización a infección en pacientes expuestos que podrían precisar de una hospitalización posterior. El consumo de antibióticos estuvo asociado con la infección en el $75 \%$ de estos pacientes, y las fluoroquinolonas son el grupo de antibióticos más prescrito (20).

La mayoría de vasculitis leucocitoclásticas son autolimitadas, ya que el $90 \%$ de los casos se dan en el transcurso de semanas a meses (10). El tratamiento depende de dos factores: su etiología y la extensión de la enfermedad. Si su causa es infecciosa, se debería tratar la infección de base. Cuando se manifiesta como parte de una vasculitis sistémica, el tratamiento de basa en la gravedad de la afectación orgánica. Generalmente, amerita el uso de esteroides e inmunosupresores (7).

En los pacientes con vasculitis leucocitoclástica y $\mathrm{CU}$ hay diferentes esquemas con base en la actividad y gravedad de la enfermedad. La enfermedad con actividad leve a moderada se trata generalmente con aminosalicilatos orales o tópicos o, a su vez, con glucocorticoides. Estos medicamentos inhiben la producción de citocinas y otros mediadores inflamatorios. Por una parte, los glucocorticoides han demostrado eficacia en inducir la remisión de la enfermedad. Sin embargo, el paciente puede presentar efectos secundarios como ganancia de peso, hiperglucemia, acné, hirsutismo, hipertensión y resorción ósea que requiere la prescripción de calcio y vitamina $\mathrm{D}$ suplementarios. En cuanto a los aminosalicilatos, se ha destacado la mesalazina. Los efectos secundarios de estos incluyen cefalea, dolor abdominal, náuseas, vómitos, diarrea, entre otros.

La enfermedad con actividad grave se caracteriza por presentar más de 6 deposiciones diarias sanguinolentas, anemia y descompensación hemodinámica. Generalmente, implican la hospitalización del paciente para tratamiento y estabilización. Cuando el tratamiento con glucocorticoides no es efectivo, se ha demostrado el uso eficaz de infliximab o ciclosporina como terapia de rescate por aproximadamente 5 a 7 días hasta demostrar su respuesta (21).

En cuanto al tratamiento a la asociación de $C$. difficile, se consideran medidas generales la suspensión de tratamientos médicos que incluyan antiperistálticos y laxantes, y la evaluación de posible suspensión de tratamientos antibióticos, opioides e inhibidores de la bomba de protones (IBP) (22).

Se debe evaluar la gravedad de la infección. En un primer episodio, si esta es leve a moderada, se administra metronidazol vía oral por 10 días. Si la infección es grave, se requiere la administración de vancomicina vía oral a dosis de $125-500 \mathrm{mg} 4$ veces al día por 14 días o fidaxomicina 200 mg 2 veces al día vía oral por 10 días. Si la infección es grave y complicada, se indica vancomicina 4 veces al día, sea por vía oral, nasoenteral o rectal como enema de retención, en conjunto con metronidazol intravenoso, ambos por un período de 14 días. Por otra parte, si la infección es recurrente, se indica el mismo esquema del primer episodio o esquemas de vancomicina en pulsos con dosis en disminución. Se indica también el trasplante de microbiota fecal a la tercera o más recurrencias (23). El trasplante de microbiota fecal ha tenido una buena respuesta clínica en el tratamiento del paciente con infección recurrente. En un estudio aleatorio se demostró la resolución de síntomas en un $94 \%$ de pacientes que recibieron vancomicina por 5 días, seguida por uno o dos trasplantes (24). Los tratamientos mencionados son los recomendados de acuerdo con múltiples ensayos clínicos. 
De las condiciones ya estudiadas se ha comprobado que la incidencia de la infección por $C$. difficile es de $1,8 \%$ a $5,7 \%$ en pacientes con CU (25). Además, la infección por C. difficile altera el curso de la CU al activar la respuesta inmunitaria hacia el patógeno que causa la infección (25). Ambas enfermedades concomitantes están relacionadas con un mayor índice de mortalidad (16).

\section{CONCLUSIONES}

La vasculitis leucocitoclástica y CU asociada a $C$. difficile es poco común y clínicamente suelen ser sincrónicas en su aparición, aunque la aparición de lesiones cutáneas no siempre guarda relación con la actividad de la enfermedad inflamatoria intestinal. En nuestro caso, la vasculitis coincidió con agudización de la enfermedad inflamatoria intestinal que no estaba diagnosticada asociada con la infección por C. difficile. La aparición de cualquier manifestación dermatológica debe investigarse ya que llegar al diagnóstico de vasculitis puede tener importantes implicaciones que podrían repercutir en la evolución de la enfermedad inflamatoria intestinal y su mejoría clínica, así como de la vasculitis.

\section{Conflictos de interés}

No existen conflictos de intereses.

\section{Fuente de financiación}

Propias de los autores.

\section{REFERENCIAS}

1. Casano VJ, Fernández Carballido C, Ibero Díaz I, Contreras Blasco MA. Vasculitis Leucocitoclásticas. En: Román JA, Rosas JC, Castellano JA (editores). Enfermedades Reumáticas. Sociedad Valenciana de Reumatología; 2008. p. 289-299.

2. Hong JS, Jin SP, Choi M, Lee KL, Lee JH, Cho S. Annular leukocytoclastic vasculitis in a patient with ulcerative colitis. Ann Dermatol. 2011;23(Suppl 3):S338-40. https://doi.org/10.5021/ad.2011.23.S3.S338

3. Butts GT, Bishop PR, Wyatt JP, Nowicki MJ. Leukocytoclastic vasculitis in an adolescent with ulcerative colitis: Report of a case and review of the literature. SAGE Open Med Case Rep. 2014;2:2050313X14547609. https://doi.org/10.1177/2050313X14547609

4. Piche T, Vanbiervliet G, Pipau FG, Dainese R, Hébuterne $\mathrm{X}$, Rampal P, Collins SM. Low risk of irritable bowel syndrome after Clostridium difficile infection. Can J Gastroenterol. 2007;21(11):727-31. https://doi.org/10.1155/2007/262478

5. Vieira Teixeira F, Saad Hosne R, Sobrado CW.Management of ulcerative colitis: a clinical update. 4,2015 , J Coloproctol. 2015;35(04):230-7. https://doi.org/10.1016/j.jcol.2015.08.006

6. Davis SC, Robinson BL, Vess J, Lebel JS. Primary care management of ulcerative colitis. Nurse Pract. 2018;43(1):11-19. https://doi.org/10.1097/01.NPR.0000527565.05934.14

7. Gota CE, Calabrese LH. Diagnosis and treatment of cutaneous leukocytoclastic vasculitis. Int J Clin Rheumatol. 2013;8(1):49-60. https://doi.org/10.2217/ijr.12.79

8. Liste Rodríguez S, Chamizo Cabrera MG, Paz Enrique LE; Hernández Alfonso EA. Vasculitis leucocitoclástica. Revista Cubana de Medicina General Integral. 2013;30(4):415-420.
9. Langan RC, Gotsch PB, Krafczyk MA, Skillinge DD. Ulcerative colitis: diagnosis and treatment. Am Fam Physician. 2007;76(9):1323-30.

10. Baigrie D, Bansal P, Goyal A, Crane JS. Leukocytoclastic Vasculitis. 2020 Aug 11. In: StatPearls [Internet]. Treasure Island (FL): StatPearls Publishing; 2021.

11. Cerezo Ruiz A, Lenzano Grande F, Pérez De Luque DJ, Domínguez Jiménez JL, Rodríguez-Mancheño AL, Cortés Rodríguez B, Serrano Muñoz A. Vasculitis leucocitoclástica asociada a colitis ulcerosa. Gastroenterol Hepatol. 2010;33(9):682-3. https://doi.org/10.1016/j.gastrohep.2010.03.002

12. Callen JP. Severe cutaneous vasculitis complicating ulcerative colitis. Arch Dermatol. 1979;115(2):226-7.

13. Einhorn J, Levis JT. Dermatologic Diagnosis: Leukocytoclastic Vasculitis. Perm J. 2015;19(3):77-8. https://doi.org/10.7812/TPP/15-001

14. Agrawal D, Rukkannagari S, Kethu S. Pathogenesis and clinical approach to extraintestinal manifestations of inflammatory bowel disease. Minerva Gastroenterol Dietol. 2007;53(3):233-48.

15. Leukocytoclastic Vasculitis [internet]. American Osteopathic College of Dermatology [citado el 9 de septiembre de 2019]. Disponible en: https://www.aocd.org/ page/LeukocytoclasticVas

16. Rubin DT, Ananthakrishnan AN, Siegel CA, Sauer BG, Long MD. ACG Clinical Guideline: Ulcerative Colitis in Adults. Am J Gastroenterol. 2019;114(3):384-413. https://doi.org/10.14309/ajg.0000000000000152

17. Plaza Santos R, Jaquotot Herranz M, Froilán Torres C, Poza Cordón J, Casado Verrier B, de Tena Díaz-Agero FL, Vázquez López P, Suárez de Parga JM. Vasculitis leucocitoclástica asociada a enfermedad de Crohn. Gastroenterol Hepatol. 2010;33(6):433-5. https://doi.org/10.1016/j.gastrohep.2009.07.004 
18. Rodríguez-Pardo D, Mirelis B, Navarro F. Infecciones producidas por Clostridium difficile. Enferm Infecc Microbiol Clin. 2013;31(4):254-63.

https://doi.org/10.1016/j.eimc.2012.12.010

19. Khan FY, Elzouki AN. Clostridium difficile infection: a review of the literature. Asian Pac J Trop Med. 2014;7S1:S6-S13. https://doi.org/10.1016/S1995-7645(14)60197-8

20. Zacharioudakis IM, Zervou FN, Shehadeh F, Mylona EK, Mylonakis E. Association of Community Factors with Hospital-onset Clostridioides (Clostridium) difficile Infection: A Population Based U.S.-wide Study. EClinicalMedicine. 2019;8:12-19. https://doi.org/10.1016/j.eclinm.2019.02.001

21. Ford AC, Moayyedi P, Hanauer SB. Ulcerative colitis. BMJ. 2013;346:f432. https://doi.org/10.1136/bmj.f432

22. Institut national d'excellence en santé et en services sociaux (INESSS) [Internet]. Treatment of Clostridium difficile-
Associated Diarrhea or Colitis. Quebec: INESSS; 2017. Disponible en: https://www.inesss.qc.ca/fileadmin/doc/ INESSS/Rapports/Traitement/Guide_Cdifficile-EN.pdf

23. Lital Meyer S, Espinoza AR, Quera PR. Infección por clostridium difficile: epidemiología, diagnóstico y estrategias terapéuticas. Revista Médica Clínica Las Andes. 2014;25(3):473-84. https://doi.org/10.1016/S0716-8640(14)70064-1

24. Bagdasarian N, Rao K, Malani PN. Diagnosis and treatment of Clostridium difficile in adults: a systematic review. JAMA. 2015;313(4):398-408. https://doi.org/10.1001/jama.2014.17103

25. Seicean A, Moldovan-Pop A, Seicean R. Ulcerative colitis worsened after Clostridium difficile infection: efficacy of infliximab. World J Gastroenterol. 2014;20(17):5135-40. https://doi.org/10.3748/wjg.v20.i17.5135 